\title{
U-Net Based Lung Image Segmentation for Lung Disease Detection
}

\author{
Eusebio L. Mique, Alvin R. Malicdem
}

\begin{abstract}
Lung diseases are becoming a worldwide health problem. World Health Organization estimates that by 2030, lung diseases such as Chronic Obstructive Pulmonary Disease will be one of the leading cause of mortality. Accurate and timely detection of lung diseases may prevent further death. It is therefore vital that its early detection will lead to treatment and prevention of mortality among patients. However, the scarcity of expert or well-trained radiologists reading CXR images might delay the timely diagnosis of lung diseases especially in rural areas where the scarcity is felt. In order to aid radiologist in reading CXR images, a computer aided tool is proposed for faster and more accurate reading of CXR images. To prepare the image for processing, it need to be segmented to make it easier for the computer to understand. The goal of image segmentation in medical field is to extract the region of interest in the organ. This study is focused on developing a model that will segment the lung from CXR images. Using U-Net architecture based semantic segmentation, the researchers were able to develop and train a model using a set of 562 CXR images and lung mask images, 70 percent of the images were used for training and 30 percent for testing. The developed model achieved a final training accuracy of 97.55 percent and validation accuracy of 97.37 percent. Validation loss and training loss are also low which indicates that the model can segment lung from CXR images with minimal error. The developed model can then be used in classifying lung diseases by focusing on the segmented image rather than focusing on the entire CXR image.
\end{abstract}

Keywords-Image Segmentation, Lung Disease, Deep Learning, U-Net, Convolutional Neural Network

\section{INTRODUCTION}

Lung disease are disorders affecting the lungs which include asthma, chronic obstructive pulmonary (COPD) disease, tuberculosis, influenza, lung cancer, pneumonia and other breathing problems. Worldwide population are affected by huge number of related lung diseases[1] and respiratory tract infections are the top cause of death and disability[2 World Health Organization estimates that by 2030, lung diseases such as Chronic Obstructive Pulmonary Disease will be one of the leading cause of mortality. Timely treatment and to prevent further death, accurate and timely diagnosis of pulmonary disease is needed [1]. Hence, to cure the disease, its detection in its earlier phase is essential[3]. Often, X-Ray specifically chest X-Ray is the first procedure patients' undergo if doctors suspect lung disease. This is due to the fact that it is economical, an effective diagnostic tool and to its noninvasive characteristics in showing pathological

Revised Version Manuscript Received on 10 September, 2019.

Eusebio L. Mique, Jr, College of Information Technology, Don Mariano Marcos Memorial State University, City of San Fernando, Philippines.(Email: emique@dmmmsu.edu.ph)

Alvin R. Malicdem, College of Information Technology, Don Mariano Marcos Memorial State University, City of San Fernando, Philippines. (Email: amalicdem@dmmmsu.edu.ph). modifications. Chest X-Ray(CXR) gives in overall orientation as an underlying symptomatic examination and is particularly valuable in the conclusion of pneumonia, malignant growth and COPD [4]. By analyzing CXR image, radiologists can diagnose many lung related diseases[5] Skilled radiologists use CXR to recognize diseases, for example tuberculosis, pneumonia, interstitial lung malady, and cancer [6].

Classifying CXR irregularities is considered as a dreary task for radiologists[5]. Many studies have been conducted in the domain of Artificial Intelligence to aid radiologists in reading CXR Images. In the study conducted by Ramalho et. al, a new approach was proposed for classification of lung illnesses. CXR images were segmented using Adaptive Crisp Active Contours Models(ACACM) and a novel method for lung illness identification using feature extraction for segmented lung structure[1]. In the study of Abiyev and Ma'aitah, they demonstrated that classifying chest pathologies in CXR utilizing conventional and deep learning strategy is viable. The researchers presented Convolutional neural networks (CNN) in diagnosing chest illnesses[5]. Another study conducted by Kumar et. al,2012 focuses on finding nodules, early symptoms of cancer diseases, appearing in patient's lungs. The researchers utilized an altered watershed segmentation strategy to segregate the lung in an x-ray image[7].

For better classification of lung diseases, CXR images must be first segmented. The area of interest must be first separated from the entire image. Through segmentation, it aims to divide the image in a series of region based on the characteristics of the image that are almost constant in each of the region[8]. Lung segmentation is a vital move in developing a Computer-aided Diagnosis (CAD) system for the diagnosis of lung illnesses in radiographs[9]. The purpose of image segmentation is to extract the area or region of interest (ROI) in an image may it be automated or semi-automated procedure. Its goal in medical field is to extricate quantitative facts like morphometric data with regard to an organ of interest. There are two related tasks to be considered in segmentation problem, one is object recognition and the second is object delineation. In object recognition, the location of the target object on the image is determined. Object delineation on the other hand, draws the object's composition. Object recognition is a high-level procedure while object delineation alludes to a low-level procedure[10]. 
Many studies have been carried out on the use of image segmentation especially in the medical field. Norouzi et al. made a comparison of the different algorithm used in medical image segmentation. In their study, they categorized image segmentation into four. The categories are clustering method, region-based method, classifier method, and hybrid method. The pros and cons of each method were discussed. Each algorithm of the four methods was discussed for the examination of grey-level images. It was further stressed that image segmentation procedures can be chosen based on different parameters[11]. In the study of Christ et. al., cascading fully convolutional neural networks (CFCNs) and dense 3D conditional random fields (CRFs) were used in automatically segmenting liver in CT images. In their study, a 2 -fold cross-validation on 3DIRCAD was used in training CFCN models. Based on their findings, CFCN-based semantic liver segmentation attained a dice score of more than $94 \%$ for liver with calculation under $100 \mathrm{~s}$ per volume[12].

Deep learning is also gaining popularity in image segmentation. It has been the primary option for images segmentation, medical image segmentation in particular. Deep learning-based image segmentation has been set up as a vigorous instrument in image segmentation and has been generally used to isolate homogeneous areas as the first and basic part of diagnosis and treatment pipeline[13]. Alom et. al. proposed models for medical image segmentation. A Recurrent Convolutional Neural Network (RCNN) and Recurrent Residual Convolutional Neural Network (RRCNN), both are based on U-Net architecture. The proposed models were tried on three different standard datasets. Compared to other models, the outcomes show predominant performance on segmentation tasks [14]. In the study of Vesal, Ravikumar and Maier, they proposed a deep learning framework to segment organs-at-risk (OAR) in thoracic CT images, explicitly for the trachea, heart, aorta and esophagus. They employed in the bottleneck of U-net network an expanded convolutions and collected residual connections. The model attained a final Dice score of $91.57 \%$ on 20 not yet seen samples[15]. Numerous researches have also been conducted in segmenting the lungs from CXR images like the study conducted by Kalinovsky and Kovalev, which presented the result of the first exploratory stage of research and development on segmentation of lungs in $\mathrm{X}$-rays using deep learning methods and encoder-decoder convolutional neural network(EDCNN). The result obtained in this study concludes that EDCNN networks may be considered as a encouraging tool for lung segmentation in large-scale projects[16]. In the study of Heo et. al, U-Net was utilized to segment the lung from CXR. The segmented images were then used for the detection of tuberculosis. In training the U-Net,the researchers used 140 images with lung mask. The trained U-Net attained a mean Dice Coefficient of .9621 for 60 validation sets[17]. Considering the very small number of images in training the U-Net model, it was able to achieve high result. If the number of images can still be increased, the accuracy may still be improved.

This prompted the researchers to conduct a study that will create a model for lung segmentation based on U-net architecture to be used for lung disease detection. Specifically, the study aims to: a. identify CXR images and its equivalent lung mask to be used in training the model.

b. Develop a model based on U-Net Architecture to segment the lungs from CXR image for lung disease detection.

\section{METHODOLOGY}

\section{A. Data Collection}

The dataset used in training the model was downloaded from National Institute of Health(NIH) particularly Shenzhen hospital X-ray set. The dataset was collected by Shenzhen hospital in Shenzhen, Guangdong province, China. It contains a mixture of normal and abnormal x-rays. Another dataset was obtained from Kaggle, a manually segmented lung masks dataset for Shenzhen X-ray set which were done by teachers and students of Computer Engineering Department, Faculty of Informatics and Computer Engineering, National Technical University of Ukraine "Igor Sikorsky Kyiv Polytechnic Institute".

\section{B. U-Net Architecture}

In developing the model for lung image segmentation, the researcher utilized a deep learning technique U-Net which is based on Fully Convolutional Network(FCN) and modified to yield better segmentation even with few training images. U-Net Architecture was created for Bio Medical Image Segmentation by Olaf Ronneberger et al. [18]. Fig. 1 shows the diagram of the U-Net architecture. The structure has 2 fundamental parts, the convolutional encoding and convolutional decoding units. The convolution operations are implemented and then pursued by Rectified Linear Unit activation in the encoding and decoding parts of the network. A $2 \times 2$ max-pooling operations are carried out for the encoding or the down sampling unit. To up-sample the feature map, convolution transpose operations (de-convolution) are carried out in the decoding or the up sampling part [11]. A $1 \times 1$ convolution is utilized at the final layer to plot each feature vector to the preferred classes. U-Net merge the location information from the encoding path with the contextual information in the decoding path to acquire an overall information merging localization and context, which is essential to predict a good segmentation map.

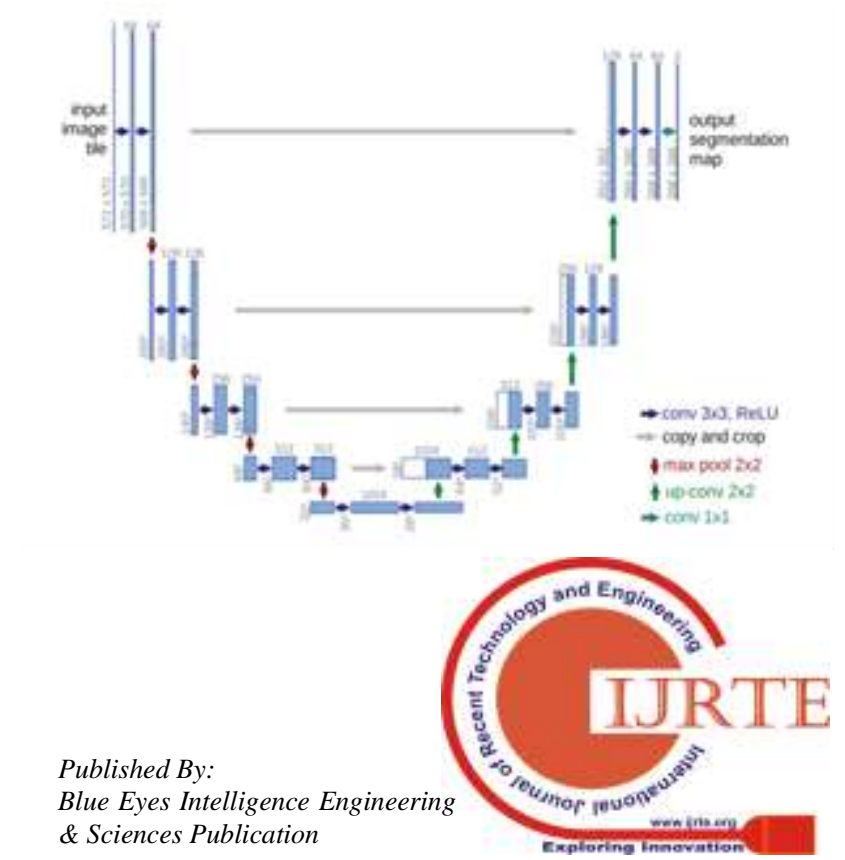


U-Net based semantic segmentation was preferred by the researchers because it does not only render better output for segmentation, it also works even with few training data [11]. Moreover, U-Net was basically created for bio medical image segmentation which is suited for this study.

\section{Training the Model}

The model in segmenting lung from CXR image was developed and trained using Python, Tensorflow and Keras. Keras is a high-level neural network API which can run on top of Tensorflow. The use of Keras allows the researchers to do fast experiments without writing large number of codes.

The training dataset consists of the original CXR images and manually segmented mask images. The dataset consists of 562 sets of mask and unmask CXR images which were divided into training and validation data. 70 percent of the CXR images were utilized for the training and 30 percent were utilized for validation. The images were resized and were trained in batches to facilitate faster training without the need of additional computing resources.

\section{RESULTS AND DISCUSSIONS}

The developed model which was based on U-Net architecture were trained using 562 sets of CXR images and manually mask images from Shenzhen No. 3 hospital. The images were resized and trained using a batch size of 32 at 40 epochs or the times the whole dataset is passed through the neural network both forward and backward path. To achieve the best possible result, the hyperparameters were adjusted until the researchers were satisfied with the outcome of the trained model.

Figure 2 shows the actual training data results produced by the python script. It shows an overall training accuracy of 97.55 percent and validation accuracy of 97.37 percent. The training loss is at .5569 and validation loss of .5291. The trained model shows a very high accuracy both on training and test images it also shows a very low training loss and validation loss which shows that the model can segment lung from unseen CXR images.
Hyperparameters

\begin{tabular}{llllll}
\hline $\begin{array}{l}\text { Training } \\
\text { Datasef } \% \text { ) }\end{array}$ & $\begin{array}{l}\text { Validation } \\
\text { Dataset(\%) }\end{array}$ & $\begin{array}{l}\text { Batch } \\
\text { Size }\end{array}$ & Epochs & $\begin{array}{l}\text { Training } \\
\text { Validation } \\
\text { Accuracy }\end{array}$ & $\begin{array}{l}\text { Training } \\
\text { Validation } \\
\text { Loss }\end{array}$ \\
\hline 80 & 20 & 8 & 25 & 96.21795 .02 & .4371 .3 \\
80 & 20 & 16 & 40 & $96.40 / 95.04$ & $.56 / 1.42$ \\
80 & 20 & 32 & 60 & $95.85 / 95.22$ & $.67 / 1.3$ \\
70 & 30 & 8 & 30 & $96.08 / 96.30$ & $.40 / 1.6$ \\
70 & 30 & 16 & 35 & $96.5 / 96.70$ & $.63 / 1.3$ \\
70 & 30 & 32 & 40 & $97.55 / 97.37$ & $.56 / 52$ \\
\hline
\end{tabular}

Table 1 shows the different training experiments conducted by the researchers in finding the best performance of the model. The researchers played with different hyperparameters and set the epocs to 1000 and observe the results of the training data. The validation set is monitored, normally, the validation loss starts decreasing, once the result starts increasing the training is terminated at the period of the smallest error. The model at the period that the training is terminated is known to provide good generalization performance. [20]. Based on the table the researchers experimented with different hyperparameter combination and monitor the results. The researchers tried splitting the dataset respectively to 80 and 20 percent training and validation data and set the batch size and epochs to different values. It can be observed that with lower batch size, the training and validation accuracy gets higher percentage. However, the difference between the validation and training loss are a bit far and did not stabilize.It can be seen from the table that when the dataset was divided into 70 and 30 percent respectivley for training and validation data and increased the batch size, the gap between the validation and training loss starts to decrease.

Fig. 3 shows a sample dataset image. The image on the left is a sample CXR image from Shenzhen Hospital and the image on the right is the manually mask CXR image done by teachers and students of National Technical University of Ukraine "Igor Sikorsky Kyiv Polytechnic Institute".

TABLE 1. Training Results with Different

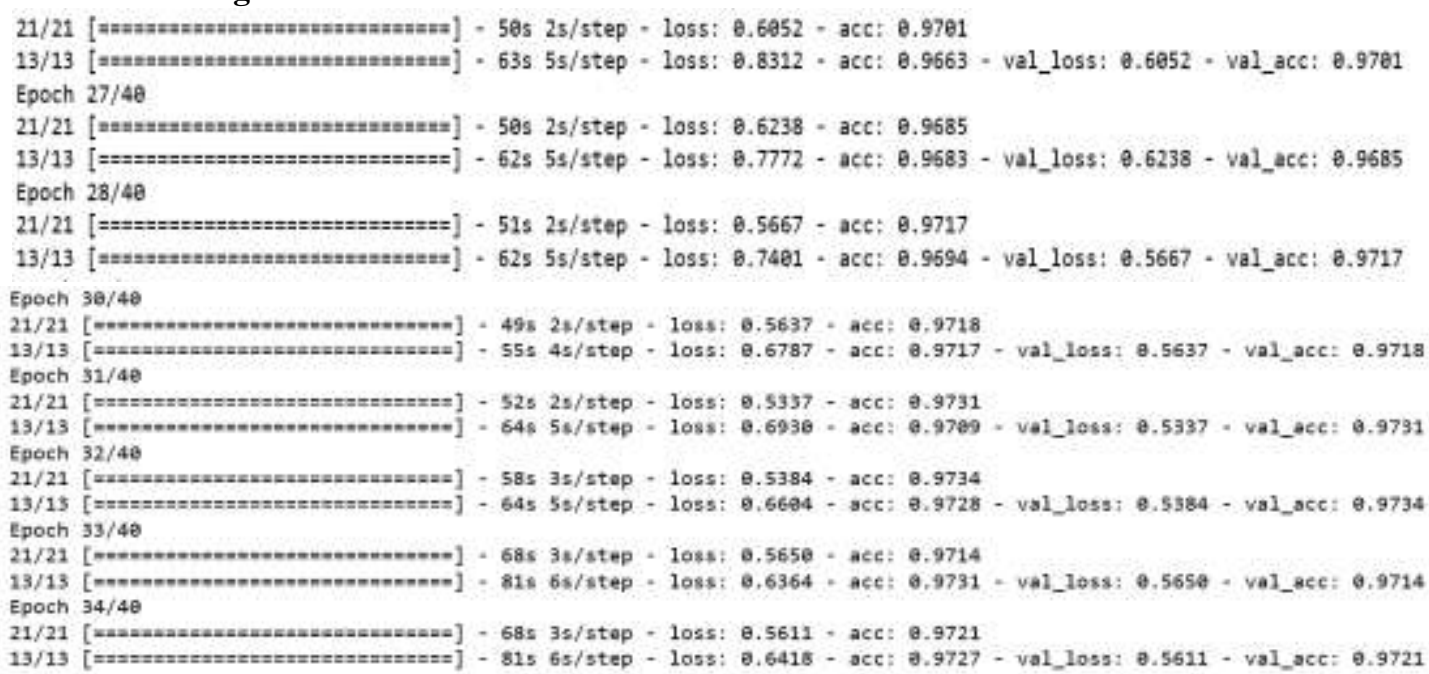




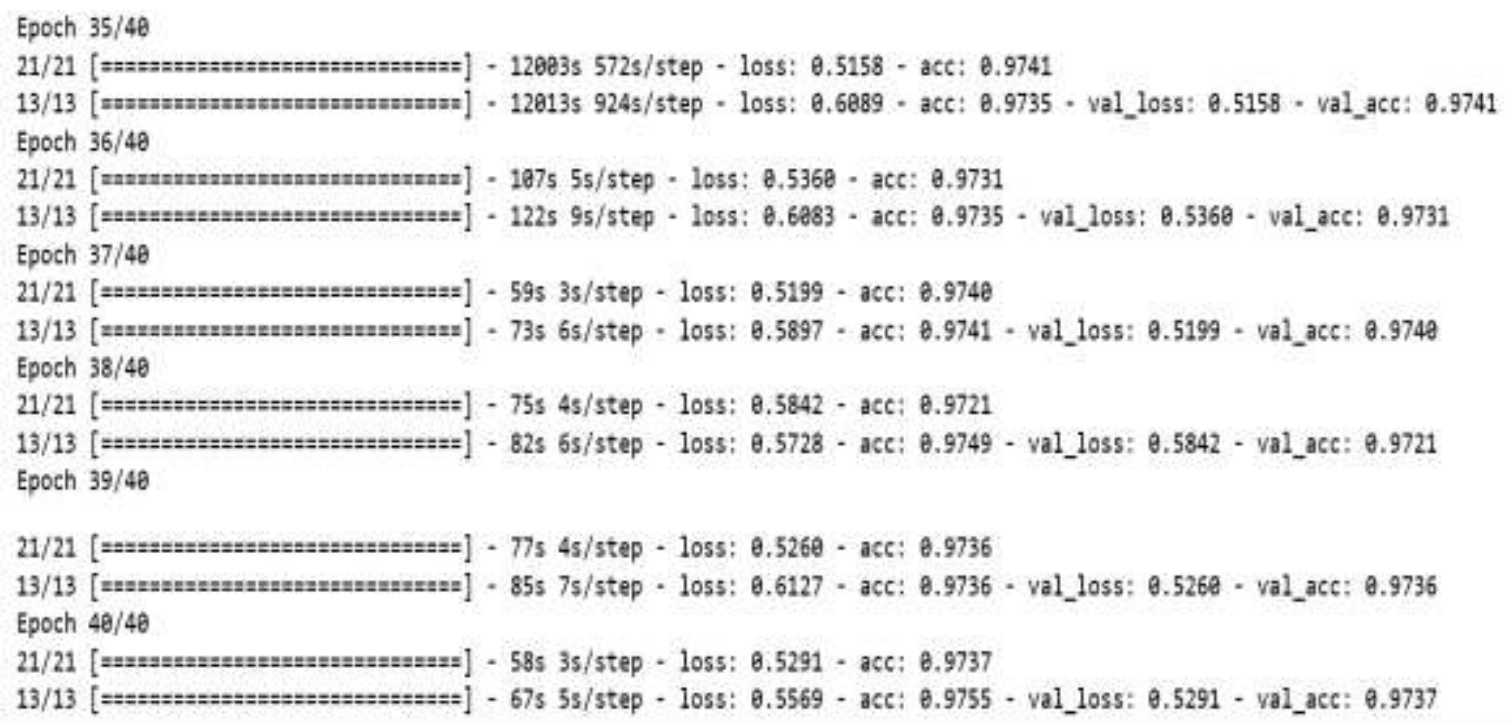

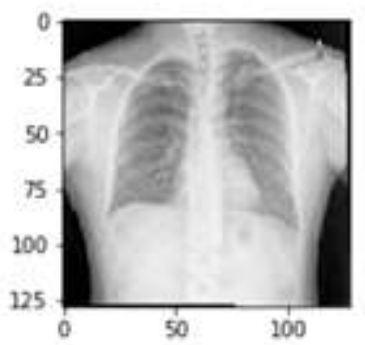

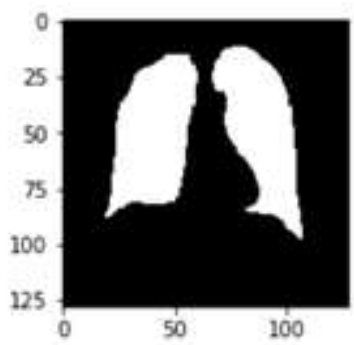

Figure 3. CXR Image Sample

Fig. 4 shows the accuracy training graph of the model. The blue line indicates the training accuracy while the orange line represents the validation accuracy. Both training and validation accuracy are high which indicates that its prediction accuracy is also high. Moreover, the validation and training loss are very low as seen in Fig 5. At around 30 epochs validation and training loss stabilized which indicates that trained model is neither overfit nor underfit. A model is said to be overfit when the train set's performance continuously improves while the validation set's performance improves at a certain point and then begins to go down. Underfit model on the other hand, is when the training dataset performs well while validation dataset performs poorly. A good fit is when both training and validation dataset performs well and it stabilized at some point [19]. Based on the graph as seen in figure 5, our model based on the result exhibited a good fit model. This indicate further that the model could predict unseen CXR images with high prediction accuracy and with very little margin of error.

Figure 6 shows sample prediction results of the developed model. The images on the left are the unseen CXR images while the images on the right are the predicted lung mask of the CXR images.

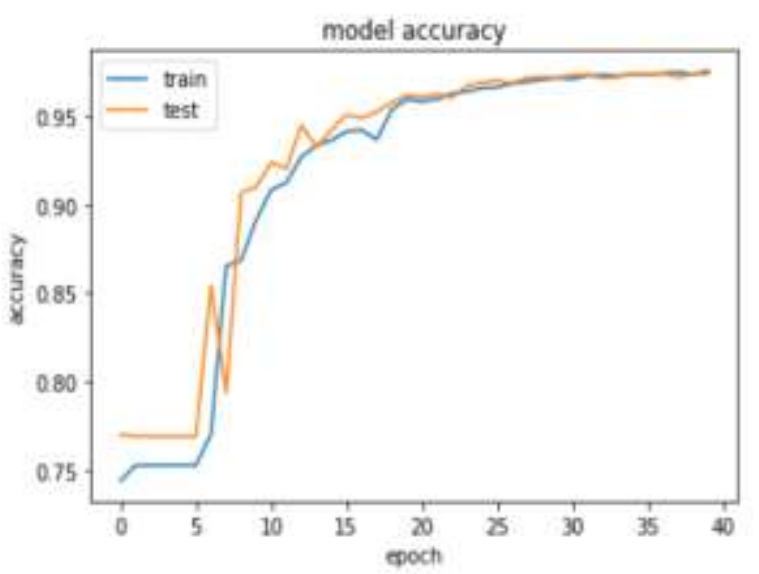

Figure 4. Training Accuracy and Validation Accuracy Graph

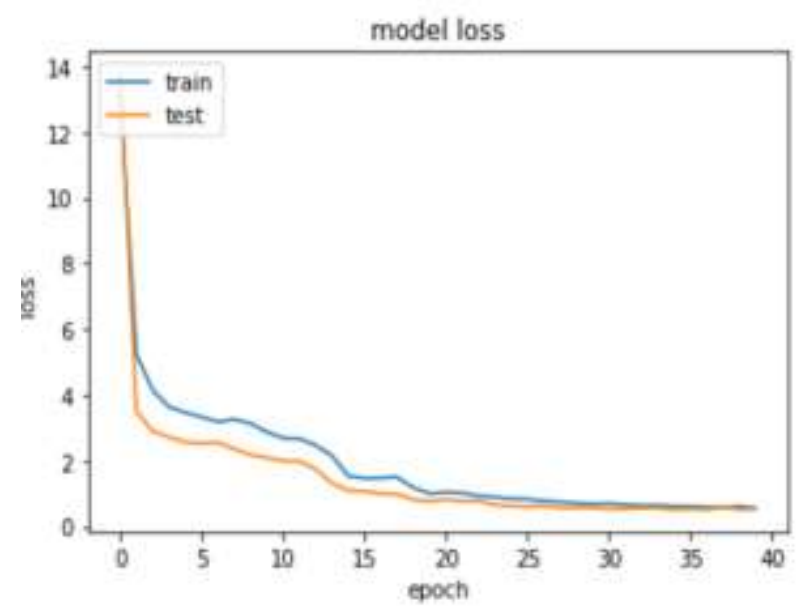

Figure 5. Training Loss and Validation Loss Graph 

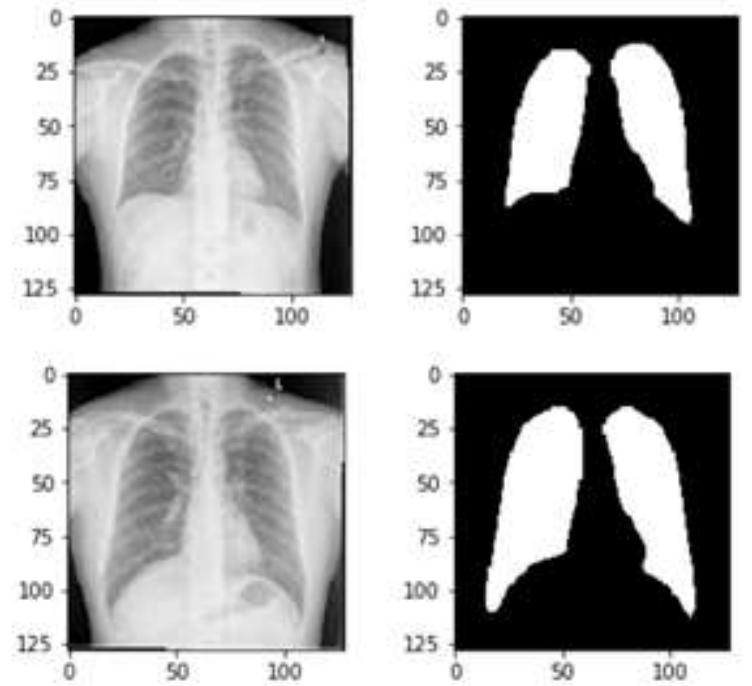

Figure 6. Sample Prediction Results

\section{CONCLUSION}

In this research study, the researchers were able to create a model that can segment the lungs from CXR images based on U-Net architecture. To precisely segment the lungs from CXR image, the model was trained using different hyperparameters to achieve the best model with high accuracy and minimal loss. 562 CXR images with manually mask lungs were used in training the model. 70 percent of the model were used as train data and the remaining 30 percent were used as testing data. The trained model was able to achieve a validation accuracy of 97.37 and a loss of .5291. Therefore, the trained model can segment the lungs from CXR images with high accuracy. The model can be used in segmenting the lungs from CXR image before predicting lung diseases as CXR includes structures other than the lungs, such as the heart and spine. These structures are not important or might hinder in detecting lung diseases[8]. Through the develop model, the classification and detection of lung diseases can be much faster as it only focuses on the lung structure rather than the entire CXR image. The findings in the study will also be useful for future researchers who will be conducting studies in the field of bio medical imaging.

\section{REFERENCES}

1 Ramalho, Geraldo L B., Rebouças Filho, P., Medeiros, Fátima N.S., Cortez, P. C. (2014). Lung disease detection using feature extraction and extreme learning machine. Revista Brasileira de Engenharia Biomédica, 30(3), 207- 14.

2 Bağcı U1, Bray M, Caban J, Yao J, Mollura DJ.Computer-assisted detection of infectious lung diseases: a review. Comput Med Imaging Graph. 2012 Jan;36(1):72-84. doi: 10.1016/j.compmedimag.2011.06.002. Epub 2011 Jul 1.

3 Singh,S.,Singh Y.,Vijay,R. Image Processing: A Cancer.International Journal of Engineering Applied Sciences and Technology, 2016 Vol. 1, Issue 3, ISSN No. 2455-2143, Pages 121-123.

4 Wielpütz MO1, Heußel CP, Herth FJ, Kauczor HU.Radiological diagnosis in lung disease: factoring treatment options into the choice of diagnostic Better Means for Diagnosis of Lung

modality. Dtsch Arztebl Int. 2014 Mar 14;111(11):181-7. doi: 10.3238/arztebl.2014.0181.

5 Abiyev, R., Ma'aitah, M K S., Deep Convolutional Neural Networks for Chest Diseases Detection. Journal of Healthcare Engineering Volume 2018, Article ID 4168538, https://doi.org/10.1155/2018/4168538

6 Qin,C., Yao,D.,Shi, Y. Song, Z. Computer-aided detection in chest radiography based on artificial intelligence: a survey. BioMedical Engineering OnLine201817:113.

https://doi.org/10.1186/s12938-018-0544-y

7 Kumar, Vinod \& Garg, Kanwal \& Kumar, Rakesh. (2012). Pulmonary Nodules Diagnosis from X-ray Imaging using Image Processing. Conference: International Conference on Recent Trends of Computer Technology in Academia

8 Stolojescu-Crisan, C., \& Holban, S. (2014). An Interactive X-Ray Image Segmentation Technique for Bone Extraction. IWBBIO.

9 Pattrapisetwong, P and Chiracharit, W. Automatic lung segmentation in chest radiographs using shadow filter and local thresholding, 2016 IEEE Conference on Computational Intelligence in Bioinformatics and Computational Biology (CIBCB), Chiang Mai, 2016, pp. 1-6. doi: 10.1109/CIBCB.2016.7758113

10 Mansoor, A., Bagci, U., Foster, B., Xu, Z., Papadakis, G. Z., Folio, L. R., ... Mollura, D. J. (2015). Segmentation and Image Analysis of Abnormal Lungs at CT: Current Approaches, Challenges, and Future Trends. Radiographics : a review publication of the Radiological Society of North America, Inc, 35(4), 1056-1076. doi:10.1148/rg.2015140232

11 Norouzi, Alireza \& Rahim, Mohd \& Altameem, Ayman \& Saba, Tanzila \& Ehsani Rad, Abdolvahab \& Rehman, Amjad \& Uddin, Mueen. (2014). Medical Image Segmentation Methods, Algorithms, and Applications. IETE Technical Review. 31 199-213. 10.1080/02564602.2014.906861.

12 Christ P.F. et al. (2016) Automatic Liver and Lesion Segmentation in CT Using Cascaded Fully Convolutional Neural Networks and 3D Conditional Random Fields. In: Ourselin S., Joskowicz L., Sabuncu M., Unal G., Wells W. (eds) Medical Image Computing and Computer-Assisted Intervention MICCAI 2016. MICCAI 2016. Lecture Notes in Computer Science, vol 9901. Springer, Cham

13 Hesamian, M.H., Jia, W., He, X. et al. "Deep Learning Techniques for Medical Image Segmentation: Achievements and Challenges" Journal of Digital Imaging (2019). https://doi.org/10.1007/s10278-019-00227-x.

14 Alom, Md. Zahangir \& Yakopcic, Chris \& Hasan, Mahmudul \& M. Taha, Tarek \& Asari, Vijayan. (2019). Recurrent residual U-Net for medical image segmentation. Journal of Medical Imaging. 6 . 10.1117/1.JMI.6.1.014006

15 Vesal, S., Ravikumar, N., \& Maier, A.K. (2019). A 2D dilated residual U-Net for multi-organ segmentation in thoracic CT. ArXiv, abs/1905.07710.M. Young, The Technical Writer's Handbook. Mill Valley, CA: University Science, 1989.

16 Kalinovsky, A., \& Kovalev, V.A. (2016). Lung Image Segmentation Using Deep Learning Methods and Convolutional Neural Networks. 
17 Heo, Seok-Jae \& Kim, Yangwook \& Yun, Sehyun \& Lim, Sung-Shil \& Kim, Jihyun \& Nam, Chung-Mo \& Park, Eun-Cheol \& Jung, Inkyung \& Yoon, Jin-Ha. (2019). Deep Learning Algorithms with Demographic Information Help to Detect Tuberculosis in Chest Radiographs in Annual Workers' Health Examination Data. International Journal of Environmental Research and Public Health. 16. 250. 10.3390/ijerph16020250.

18 O. Ronneberger, P. Fischer, T. Brox.,"U-Net: Convolutional Networks for Biomedical Image Segmentation". In: Navab N., Hornegger J., Wells W., Frangi A. (eds) Medical Image Computing and Computer-Assisted Intervention - MICCAI 2015. MICCAI 2015. Lecture Notes in Computer Science, vol 9351. Springer, Cham

19 J. Brownlee "How to Diagnose Overfitting and Underfitting of LSTM Models"[Online].Available:https://machinelearning mastery.com/diagnose-overfittingunderfitting-lstm-models/ [Accessed 19 July, 2019]

20 J. Brownlee " A Gentle Introduction to Early Stopping to Avoid Overtraining Neural Networks". [Online].Available https://machinelearningmastery.com/early-stoppingto-avoid-overtraining-neural-network-models/.

[Accessed 19 July, 2019] 